\title{
Enhancement of Radiotherapeutic Efficacy by Paclitaxel-Loaded pH-Sensitive Block Copolymer Micelles
}

\author{
Joohee Jung, ${ }^{1,2}$ Min Sang Kim, ${ }^{3}$ Sung-Jin Park, ${ }^{1,4}$ Hye Kyung Chung, ${ }^{5}$ Jinhyang Choi, ${ }^{1}$ \\ Jaesook Park, ${ }^{1}$ Dong-Hoon Jin, ${ }^{1}$ Si Yeol Song, ${ }^{1,6}$ Heon Joo Park, ${ }^{7}$ Doo Sung Lee, ${ }^{3}$ \\ Seong-Yun Jeong, ${ }^{1}$ and Eun Kyung Choi ${ }^{1,5,6}$ \\ ${ }^{1}$ Institute for Innovative Cancer Research, Asan Medical Center, University of Ulsan College of Medicine, \\ Seoul 138-736, Republic of Korea \\ ${ }^{2}$ College of Pharmacy, Duksung Women's University, Seoul 132-714, Republic of Korea \\ ${ }^{3}$ Theranostic Macromolecular Research Center, Department of Polymer Science and Engineering, Sungkyunkwan University, Suwon \\ 440-746, Republic of Korea \\ ${ }^{4}$ Laboratory of Bioimaging Probe Development, Singapore Bioimaging Consortium, Singapore 138667 \\ ${ }^{5}$ Center for Development and Commercialization of Anti-Cancer Therapeutics, Asan Medical Center, Seoul 138-736, Republic of Korea \\ ${ }^{6}$ Department of Radiation Oncology, Asan Medical Center, University of Ulsan College of Medicine, Seoul 138-736, Republic of Korea \\ ${ }^{7}$ Department of Microbiology, College of Medicine, Inha University, Inchon 400-712, Republic of Korea
}

Correspondence should be addressed to

Seong-Yun Jeong, syj@amc.seoul.kr and Eun Kyung Choi, ekchoi@amc.seoul.kr

Received 13 February 2012; Accepted 31 March 2012

Academic Editor: Haifeng Chen

Copyright ( 92012 Joohee Jung et al. This is an open access article distributed under the Creative Commons Attribution License, which permits unrestricted use, distribution, and reproduction in any medium, provided the original work is properly cited.

Radiotherapy (RT) is a major modality for cancer treatment, but its efficacy is often compromised by the resistance caused by tumor-specific microenvironment including acidosis and hypoxia. For an effective RT, concurrent administration of radiosensitizer with RT has been emphasized. However, most anticancer agents enhancing radiotherapeutic efficacy have obstacles such as poor solubility and severe toxicity. Paclitaxel (PTX), a well-known radiosensitizer, is insoluble in water and needs toxic solvent like Cremophor EL. Nanomaterials in drug delivery systems have been utilized for improving the drawbacks of anti-cancer drugs. Solubilization, tumor accumulation, and toxicity attenuation of drug by nanomaterials are suitable for enhancement of radiotherapeutic efficacy. In this study, PTX was incorporated into $\mathrm{pH}$-sensitive block copolymer micelle (psm-PTX), polyethylene glycol-graft-poly $(\beta$-amino ester), and preclinically evaluated for its effect on RT. The size of psm-PTX was $125.7 \pm 4.4 \mathrm{~nm}$ at pH 7.4. psm-PTX released PTX rapidly in the acidic condition ( $\mathrm{pH}$ 6.5), while it was reasonably stable in the physiologic condition ( $\mathrm{pH}$ 7.4). The clonogenic assay showed that psm-PTX greatly sensitized human non-small-cell lung cancer A549 cells to radiation. In the xenograft tumor model, the combination of psm-PTX and radiation significantly delayed the tumor growth. These results demonstrated the feasibility of psm-PTX to enhance the chemoradiotherapeutic efficacy.

\section{Introduction}

Radiotherapy (RT) is a major treatment for many cancers, especially non-small-cell lung cancer (NSCLC), but the overall cure rate and survival rate remain low because of radioresistance by proficient repair of radiation damage, accelerated cell repopulation, hypoxia, various molecular inhibitors of cell death, and so on [1]. To increase tumor control, concurrent administration of radiosensitizer with
RT has been a common treatment regimen [2]. Most radiosensitizers are mainly anticancer agents. One of well known radiosensitizers is paclitaxel (PTX), which is being widely studied for clinical trials [3]. However, PTX needs toxic solvent such as Cremophor EL for clinical application due to its insolubility and has toxicities such as neutropenia, peripheral sensory neuropathy, dermatitis, and hypersensitivity reaction. Thus, there has been passionate interest in developing alternative formulation of PTX. 
Nanomaterials in drug delivery systems (DDSs) have been playing a key role in the medical field, especially anticancer therapy. Nanomaterials such as polymeric nanoparticles and micelles are associated with solubilization of lowsolubility drugs. PTX-loaded NK105 micellar nanoparticles, Abraxane (PTX-loaded albumin particle), Xyota (polymerconjugated PTX), and Tocosol (tocophenol-based PTX), improve the low solubility of PTX. They are being evaluated in preclinical and clinical studies $[4,5]$. Furthermore, these nanomaterials are suitable for drug accumulation in tumor by the enhanced permeability and retention (EPR) effect. Recently many efforts have been dedicated to study modified nanomaterials with tumor cell targeting moieties or tumor environmental sensitivity (temperature, $\mathrm{pH}$, and enzyme activity). These properties control cell selectivity, cellular uptake, and drug release. Among them, tumor acidity, slightly a low extracellular $\mathrm{pH}(\sim 6.8)$ [6], is exploited for DDS. pH-sensitive nanomaterials are suited for controlled drug release by the normal/cancer cells microenvironmental difference. pH-sensitive drug carriers have been developed to include liposome, micelles, dendrimers, and drug-polymer conjugates [7-9]. In our previous study, methyl ether poly(ethylene glycol)-poly( $\beta$ amino ester) polymeric micelles (MPEG-PM) was synthesized and evaluated as a PTX-loaded smart carrier in vitro [10]. Moreover, camptothecin-incorporated MPEG-PM was reported for cancer therapeutic efficacy [11]. Herein, we evaluated PTX-loaded pH-sensitive micelle (psm-PTX) as a radiosensitizer for chemoradiotherapy in NSCLC both in vitro and in vivo.

\section{Materials and Methods}

2.1. Preparing psm-PTX. pH-sensitive block copolymer micelle (psm), of which $M_{n}$ is $13,000 \mathrm{~g} \cdot \mathrm{mol}^{-1}$ and which contains 4 mol- $\%$ PEG, was obtained according to the previous study [12]. PTX was conjugated with psm using anhydrous dichloromethane as reported previously [13]. The size distribution of psm-PTX was measured by dynamic light scattering (DLS) at $25^{\circ} \mathrm{C}$.

2.2. PTX Release from $p s m-P T X$ In Vitro. To determine the release of PTX depending on $\mathrm{pH}$, psm-PTX $(1 \mathrm{mg} / \mathrm{mL})$ solution at $\mathrm{pH}$ 6.0-8.0 was prepared. The $\mathrm{pH}$ of psm-PTX was adjusted stepwise with $\mathrm{NaOH}$ solution and immediately filtered. To measure the release of PTX depending on time, $1 \mathrm{mg} / \mathrm{mL}$ of psm-PTX was loaded to a dialysis membrane (MWCO, 3500) and immersed in $20 \mathrm{~mL}$ of the medium at $37^{\circ} \mathrm{C}$. At predetermined time intervals, the medium was withdrawn. The concentration of PTX was measured by HPLC as described in the previous study [12]. Briefly, the mobile phase was changed linearly from acetonitrilewater $(50: 50 \mathrm{v} / \mathrm{v})$ to acetonitrile-water $(90: 10 \mathrm{v} / \mathrm{v})$, and a C-18 reverse-phase column was used. The flow rate was $0.5 \mathrm{~mL} / \mathrm{min}$.

2.3. Clonogenic Assay. Human lung adenocarcinoma A549 cells purchased from the American Type Culture Collection
(ATCC) were maintained in Dulbecco's modified Eagle's medium (DMEM; Invitrogen, Carlsbad, CA) supplemented with $10 \%$ fetal bovine serum (Invitrogen) and 1\% penicillin/streptomycin under a humidified atmosphere of $5 \%$ $\mathrm{CO}_{2}$ at $37^{\circ} \mathrm{C}$. A549 cells were seeded from 100 to 1,000 cells/well in 6-well plate and treated with PTX or psm-PTX $(0.2 \mathrm{nM}$ as PTX). PTX was dissolved by $50 \%$ Cremophor EL/ethanol and diluted one-fifth by phosphate-buffered saline ( $\mathrm{pH}$ 7.4) before use. After $2 \mathrm{~h}, \mathrm{~A} 549$ cells were irradiated at $2 \mathrm{~Gy}$ using a $6 \mathrm{MV}$ photon beam linear accelerator (CL/1800, Varian Medical System, Palo Alto, CA). The cells were incubated for 11 days to allow colony formation. The colonies composing of over 50 cells were counted, and the survival fraction was calculated as in the previously study [14]. Plating efficiency was defined from nonirradiated cells as (mean colonies counted)/(cells plated). Survival fraction was calculated as (mean colonies counted) $/[$ (cells plated) $\times$ (plating efficiency)].

2.4. Tumor Growth Delay. All experiments were performed following the protocol approved by the Institutional Animal Care and Use Committee of ASAN Institute for Life Science. A549 cells $\left(5 \times 10^{5}\right.$ cells $)$ were implanted subcutaneously into the right hind leg of five-week-old male $\mathrm{BALB} / \mathrm{c}$ nude mice weighing about $20 \mathrm{~g}$ (Japan SLC, Shizuoka, Japan), as previously described [15]. When the tumors grew to about $100 \mathrm{~mm}^{3}$, the mice were randomized and divided into the experimental groups ( $n=5 /$ group $)$. The mice were intravenously injected with $10 \mathrm{mg} / \mathrm{kg}$ of psm-PTX (as at a concentration of PTX) or equal amount of psm $(300 \mathrm{mg} / \mathrm{kg})$ without PTX as a control. After $2 \mathrm{~h}$, tumors were irradiated to $5 \mathrm{~Gy}$. The tumor volume was calculated by the following formula: $V=($ longest dimension $) \times(\text { shortest dimension })^{2}$ $\times 0.5$.

\section{Results and Discussions}

3.1. Characterization of $p s m-P T X$. For $\mathrm{pH}$-sensitivity and hydrophilic block, psm consisted of poly( $\beta$-amino ester) and PEG (Figure 1(a)). Molecular weight of PEG was 2,000, which was known to be degradable without toxicity $[16,17]$. Furthermore, the micellization/demicellization transition of psm was reported at the sharp $\mathrm{pH}$ transition between 6.4 and 6.8 in previous work [11]. PTX was easily loaded into psm without an organic solvent. Loading efficiency of PTX was $48 \%$, and loading amount was $2.3 \%(\mathrm{w} / \mathrm{w})$. Lyophilized psmPTX was reconstructed by sterilized water for the evaluation of psm-PTX. As a result, psm-PTX improved the water solubility of PTX by hydrophilic block, suggesting it excluded cremophor EL-induced toxicity. The size of psm-PTX was determined as $125.7 \pm 4.4 \mathrm{~nm}$ at $\mathrm{pH} 7.4$ by DLS, while psm was $27.7 \pm 1.2 \mathrm{~nm}$ (Figure 1(b)). The encapsulation of PTX in psm expanded the size of polymeric micelles. These results suggested that the properties of psm-PTX (its size, solubility, and $\mathrm{pH}$ sensitivity) could play a smart carrier role in chemotherapy.

3.2. $p H$-Dependent Drug Release of psm-PTX. psm-PTX was investigated for controlled drug release. psm-PTX showed 


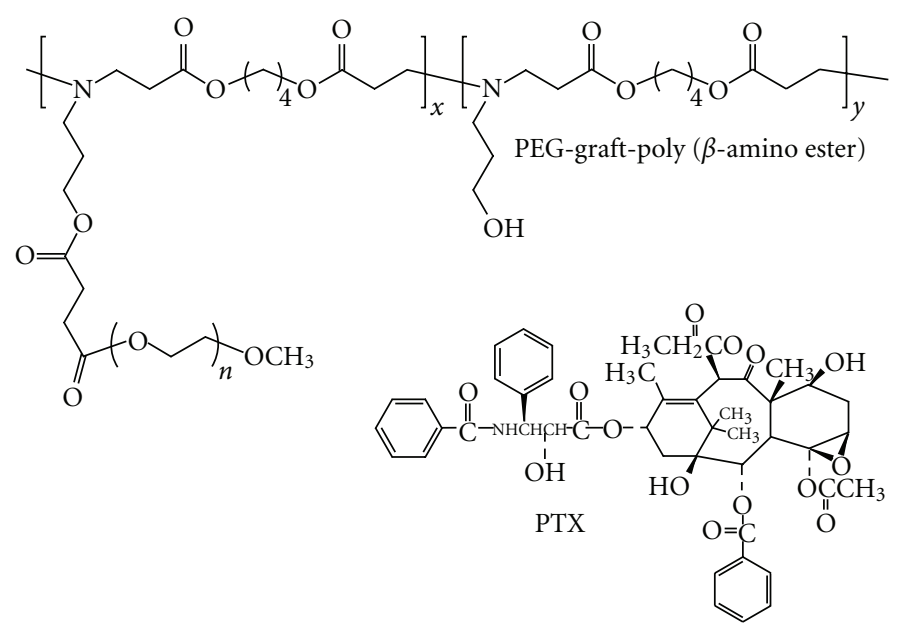

(a)

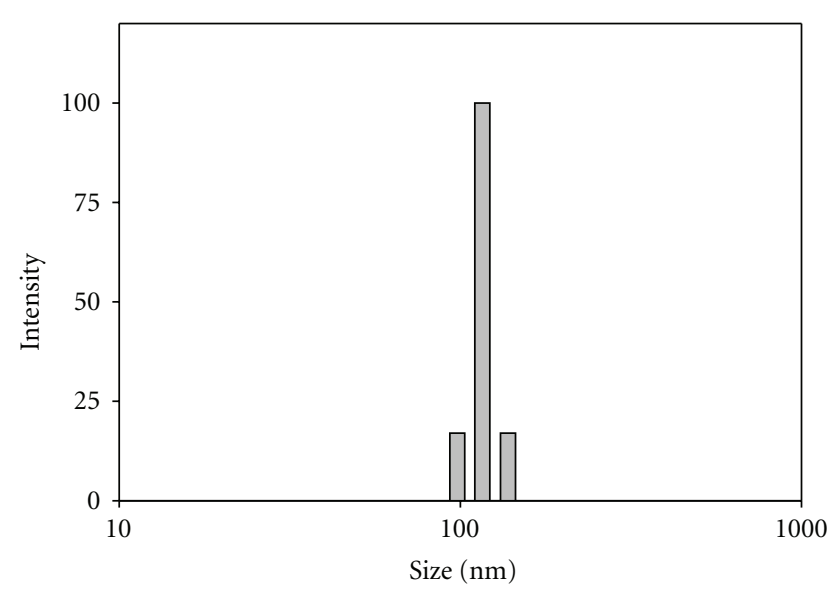

(b)

Figure 1: Composition (a) and size (b) of psm-PTX.

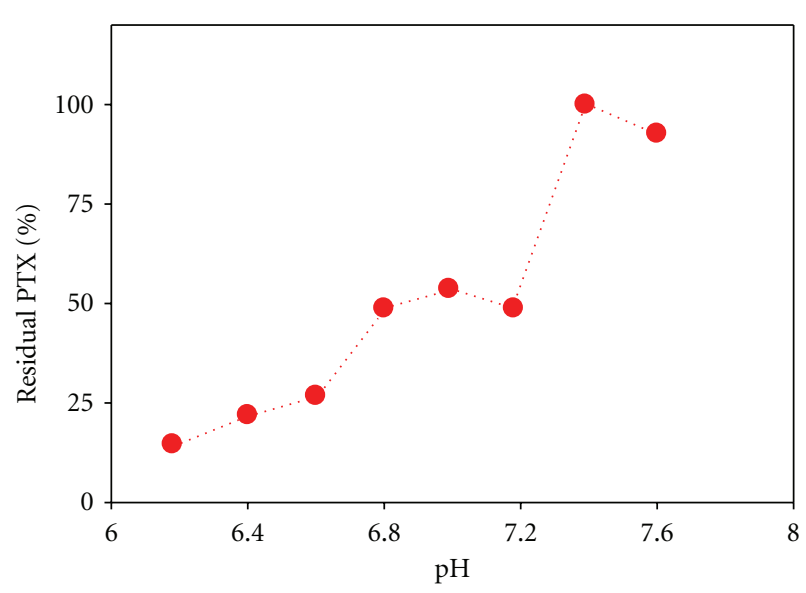

(a)

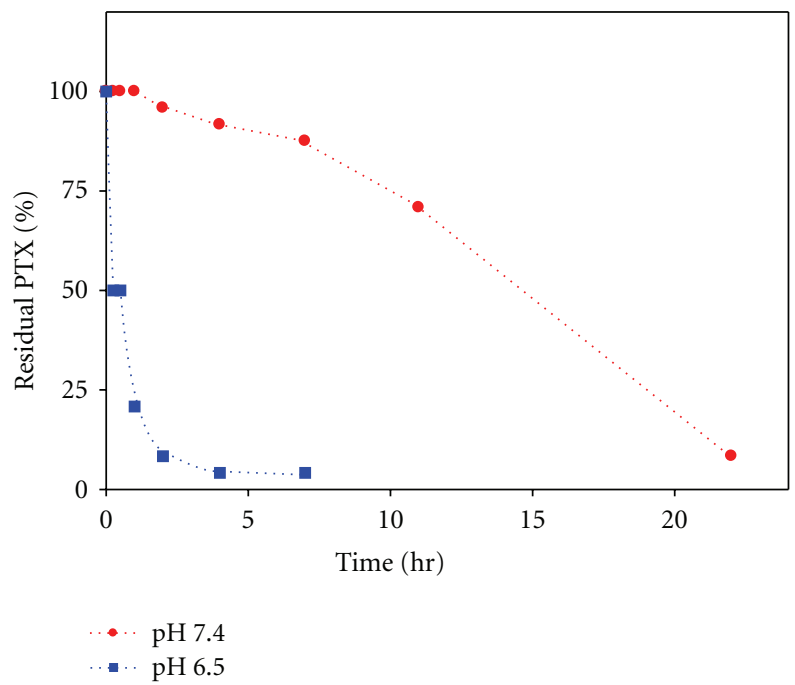

(b)

Figure 2: In vitro release of PTX from psm-PTX. (a) pH-dependent PTX release. psm-PTX (25 $\mu \mathrm{g} / \mathrm{ml}$ as PTX) was immediately filtered after titration. (b) Time-dependent PTX release. psm-PTX $\left(25 \mu \mathrm{g} / \mathrm{ml}\right.$ as PTX) was filtered after incubation at $37^{\circ} \mathrm{C}$. PTX was analyzed by HPLC as described in Section 2.

a $\mathrm{pH}$-dependent drug release according to the different $\mathrm{pH}$ conditions (Figure 2(a)). Below pH 6.8, PTX was dramatically released over $50 \%$ from psm-PTX. Moreover, $50 \%$ of PTX in psm-PTX was rapidly released PTX within $30 \mathrm{~min}$ and then psm-PTX remained less $10 \%$ PTX at $4 \mathrm{~h}$ under the $\mathrm{pH} 6.5$ condition, indicating that psm-PTX was demicellized at the acidic $\mathrm{pH}$ (Figure 2(b)). The results suggested that psm-PTX could trigger the PTX release at tumor site due to the acidic environment of the tumor site. On the other hand, psm-PTX was stable and slowly released for $12 \mathrm{~h}$ at $37^{\circ} \mathrm{C}$ in $\mathrm{pH} 7.4$ (Figure 2(b)), suggesting that psm-PTX preserved PTX during systemic circulation. However, residual PTX did not remain for a day at normal $\mathrm{pH}$, and thus the modified formulation of psm-PTX is being studied for a long circulation.

3.3. Radiosensitizing Effect of psm-PTX in A549 Human Lung Cancer Cells. The radiosensitizing effect of psm-PTX was evaluated on A549 cells by clonogenic assay (Figure 3). Survival fraction of psm did not differ from control at any $\mathrm{pH}$. Survival fraction of PTX was similar to that of ionizing radiation (IR, 2Gy), and the combination of PTX with IR showed the expectative sensitizing effect. Importantly, psm-PTX showed greatly lower survival fraction than PTX, suggesting that psm-PTX was uptaken rapidly into cells and 


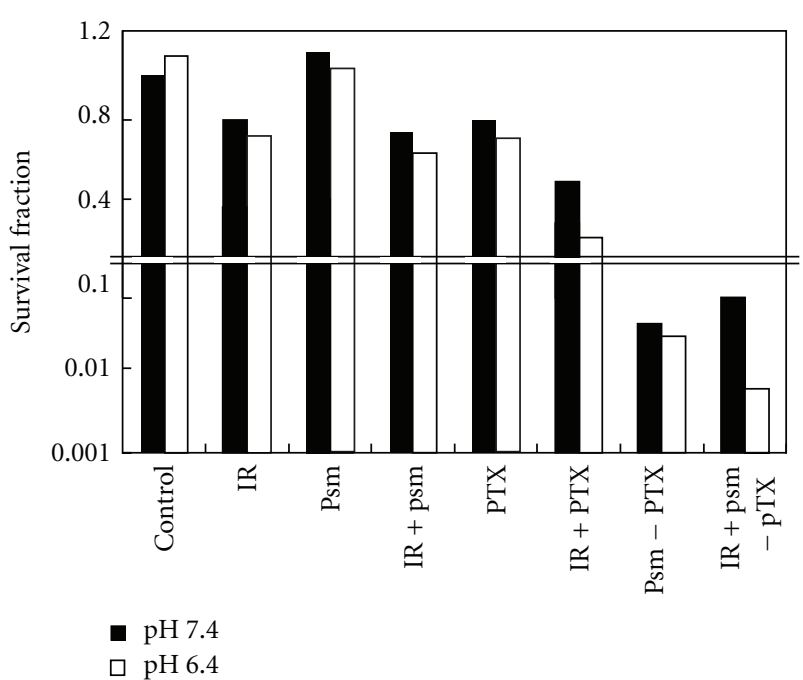

FIGURe 3: In vitro radiosensitizing effect of psm-PTX. A549 cells were treated with PTX or psm-PTX $(0.2 \mathrm{nM}$ as PTX) for $2 \mathrm{~h}$ and irradiated at $2 \mathrm{~Gy}$. After 11 days, colony was counted and survival fraction was calculated.

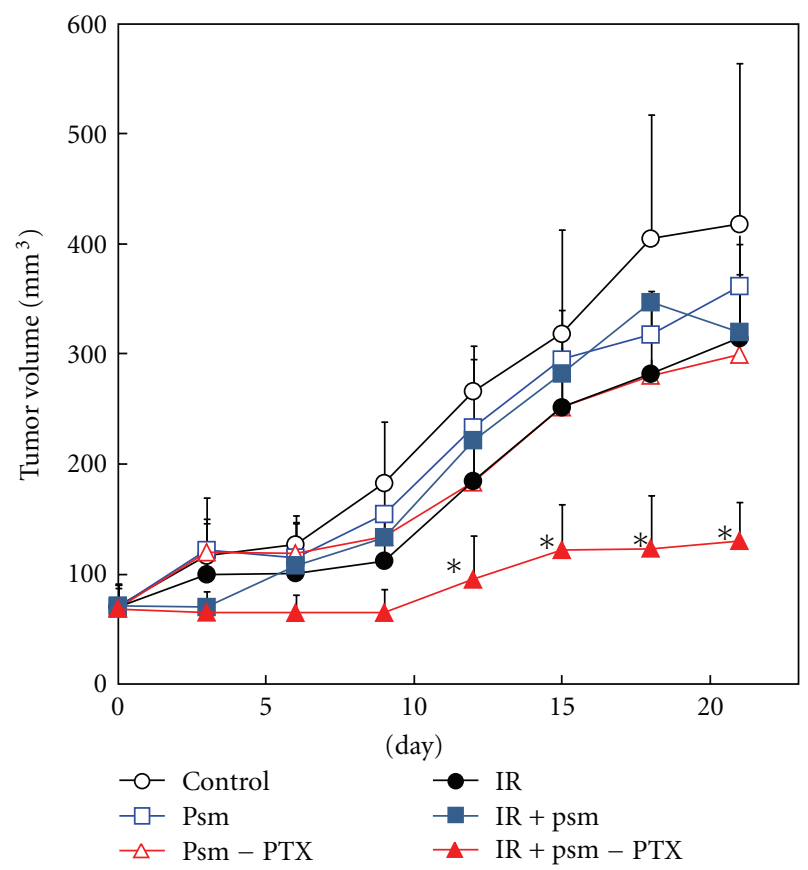

FIGURe 4: In vivo tumor growth delay of psm-PTX. Mice bearing A549-derived tumor were treated with PTX or psm-PTX $(10 \mathrm{mg} / \mathrm{kg}$ as PTX) and irradiated at $5 \mathrm{~Gy}$ after $2 \mathrm{~h}$. Tumor size was measured every 3 days and tumor volume was calculated as in Section 2. Data present mean \pm standard deviation $\left({ }^{*}, P<0.05, t\right.$-test $)$.

internalized psm-PTX was demicellized by the endosomal acid $\mathrm{pH}$ (below $\mathrm{pH}$ 6.5). Especially, the combination of psmPTX with IR showed more cytotoxicity than psm-PTX or IR in the low acidic $\mathrm{pH}$ environment ( $\mathrm{pH}$ 6.5). These results showed that psm-PTX had a potential to exert an enhanced radiosensitization effect at the acidic $\mathrm{pH}$ condition.
3.4. In Vivo Tumor Growth Delay of the Combination of psm-PTX with IR. The acidic environment of the tumor is an important property for active targeting. In the previous study, the tumor growth delay of anticancer drug was enhanced by using camptothecin- (CPT-) loaded $\mathrm{pH}-$ responsive polymeric micelles (CPT-pH-PMs) [11]. In this study, the radiotherapeutic efficacy of psm-PTX was evaluated in mice bearing A549-derived tumor. The psm-PTX was administered by intravenous injection via tail vein. As shown in Figure 4, psm-PTX-treated group showed similar tumor growth to IR-treated group. The combination of psmPTX with IR significantly enhanced the radiotherapeutic efficacy. At 21 days after administration, psm-PTX, IR, and the combination of psm-PTX with IR suppressed the tumor size by $71 \%, 75 \%$, and $31 \%$, respectively. During this examination, the change of body weight was not shown in any group. The results indicated that psm-PTX as a radiosensitizer was able to be utilized for chemoradiotherapy.

Chemoradiotherapy is a standard treatment regimen for various cancers. In addition, nanomaterials as drug carriers induce the enhancement of therapeutic efficacy as well as the attenuation of toxicity. Abraxane, approved by US Food and Drug Administration, was also reported to improve radiotherapy [18]. Although several $\mathrm{pH}$-responding carriers containing PTX have been reported for chemotherapeutics, an utilization of $\mathrm{pH}$-responding carrier as radiosensitizer has not been reported. Therefore, we aimed to apply psmPTX, a smart carrier responding the tumor-specific microenvironment such as low $\mathrm{pH}$, to improve radiotherapeutic efficacy. The results of this study demonstrated the feasibility of psm-PTX to enhance the chemoradiotherapeutic efficacy. Inasmuch as this study was designed primarily for the enhancement of concurrent chemoradiotherapy, we have focused on the radiosensitization effect in the presence of psm-PTX during radiation. Although we have not evaluated the effect of psm-PTX on a sequential chemoradiotherapy in which chemotherapy and RT are separately given to tumor, further investigation to confirm the efficacy of psm-PTX on sequential chemoradiotherapy is clearly warranted. This study suggests that psm-PTX as a radiosensitizer would be a potential candidate for clinical chemoradiotherapy trials.

\section{Acknowledgments}

This work was supported by a grant from the Korean Health Technology R\&D Project, Ministry for Health and Welfare, Republic of Korea (A062254 and A102059), the Nuclear R\&D program through the Korea Science and Engineering Foundation funded by the Ministry of Education, Science and Technology of Korea (2008-03876), the Basic Science Research Program through the National Research Foundation of Korea (NRF) funded by the Ministry of Education, Science and Technology (KRF-2008-313E00444), and a grant from ASAN Institute for Life Science, Seoul, Republic of Korea (2010-445). D. S. Lee. would like to thank the support by the Basic Science Research Program through a National Research Foundation of Korea grant funded by the Korean Government (MEST) (2010-0027955). 


\section{References}

[1] W. T. Sause, C. Scott, S. Taylor et al., "Radiation Therapy Oncology Group (RTOG) 88-08 and Eastern Cooperative Oncology Group (ECOG) 4588: preliminary results of a phase III trial in regionally advanced, unresectable non-small-cell lung cancer," Journal of the National Cancer Institute, vol. 87, no. 3, pp. 198-205, 1995.

[2] O. Caffo, "Radiosensitization with chemotherapeutic agents," Lung Cancer, vol. 34, no. 4, supplement, pp. 81-90, 2001.

[3] D. L. Carter, D. Garfield, J. Hathorn et al., "A randomized phase III trial of combined paclitaxel, carboplatin, and radiation therapy followed by weekly paclitaxel or observation for patients with locally advanced inoperable non-small-cell lung cancer," Clinical Lung Cancer, vol. 13, no. 3, pp. 205-213, 2012.

[4] Y. Matsumura and K. Kataoka, "Preclinical and clinical studies of anticancer agent-incorporating polymer micelles," Cancer Science, vol. 100, no. 4, pp. 572-579, 2009.

[5] J. B. Bulitta, P. Zhao, R. D. Arnold et al., "Mechanistic population pharmacokinetics of total and unbound paclitaxel for a new nanodroplet formulation versus Taxol in cancer patients," Cancer Chemotherapy and Pharmacology, vol. 63, no. 6, pp. 1049-1063, 2009.

[6] M. Stubbs, P. M. J. McSheehy, J. R. Griffiths, and C. L. Bashford, "Causes and consequences of tumour acidity and implications for treatment," Molecular Medicine Today, vol. 6, no. 1, pp. 15-19, 2000.

[7] S. Salmaso, S. Bersani, M. Pirazzini, and P. Caliceti, "PHsensitive PEG-based micelles for tumor targeting," Journal of Drug Targeting, vol. 19, no. 4, pp. 303-313, 2011.

[8] D. Chen, X. Jiang, J. Liu, X. Jin, C. Zhang, and Q. Ping, "In vivo evaluation of novel $\mathrm{pH}$-sensitive mPEG-Hz-chol conjugate in liposomes: pharmacokinetics, tissue distribution, efficacy assessment," Artificial Cells, Blood Substitutes, and Biotechnology, vol. 38, no. 3, pp. 136-142, 2010.

[9] J. K. Kim, V. K. Garripelli, U. H. Jeong, J. S. Park, M. A. Repka, and S. Jo, "Novel pH-sensitive polyacetal-based block copolymers for controlled drug delivery," International Journal of Pharmaceutics, vol. 401, no. 1-2, pp. 79-86, 2010.

[10] M. S. Kim and D. S. Lee, "In vitro degradability and stability of hydrophobically modified $\mathrm{pH}$-sensitive micelles using MPEGgrafted poly( $\beta$-amino ester) for efficient encapsulation of paclitaxel," Journal of Applied Polymer Science, vol. 118, no. 6, pp. 3431-3438, 2010.

[11] K. H. Min, J. H. Kim, S. M. Bae et al., "Tumoral acidic pHresponsive MPEG-poly( $\beta$-amino ester) polymeric micelles for cancer targeting therapy," Journal of Controlled Release, vol. 144, no. 2, pp. 259-266, 2010.

[12] W. S. Shim, S. W. Kim, E. K. Choi, H. J. Park, J. S. Kim, and D. S. Lee, "Novel pH sensitive block copolymer micelles for solvent free drug loading," Macromolecular Bioscience, vol. 6, no. 2, pp. 179-186, 2006.

[13] M. S. Kim, G. H. Gao, S. W. Kang, and D. S. Lee, "Evaluation of $\mathrm{pH}$-Sensitive poly( $\beta$-amino ester)-graft-poly(ethylene glycol) and its usefulness as a pH-Sensor and protein carrier," Macromolecular Bioscience, vol. 11, no. 7, pp. 946-951, 2011.

[14] J. Jung, E.-J. Kim, H. K. Chung, H. J. Park, S.-Y. Jeong, and E. K. Choi, "c-Myc down-regulation is involved in proteasome inhibitor-mediated enhancement of radiotherapeutic efficacy in non-small cell lung cancer," International Journal of Oncology, vol. 40, no. 2, pp. 385-390, 2012.

[15] S. Y. Jeong, S. J. Park, S. M. Yoon et al., "Systemic delivery and preclinical evaluation of $\mathrm{Au}$ nanoparticle containing $\beta$-lapachone for radiosensitization," Journal of Controlled Release, vol. 139, no. 3, pp. 239-245, 2009.

[16] O. Biondi, S. Motta, and P. Mosesso, "Low molecular weight polyethylene glycol induces chromosome aberrations in Chinese hamster cells cultured in vitro," Mutagenesis, vol. 17, no. 3, pp. 261-264, 2002.

[17] A. D. Mitchell, A. E. Auletta, D. Clive, P. E. Kirby, M. M. Moore, and B. C. Myhr, "The L5178Y/tk(+/-) mouse lymphoma specific gene and chromosomal mutations assay: a phase III report of the U.S. environmental protection agency Gene-Tox program," Mutation Research, vol. 394, no. 1-3, pp. 177-303, 1997.

[18] N. Wiedenmann, D. Valdecanas, N. Hunter et al., “130-nm albumin—bound paclitaxel enhances tumor radiocurability and therapeutic gain," Clinical Cancer Research, vol. 13, no. 6, pp. 1868-1874, 2007. 

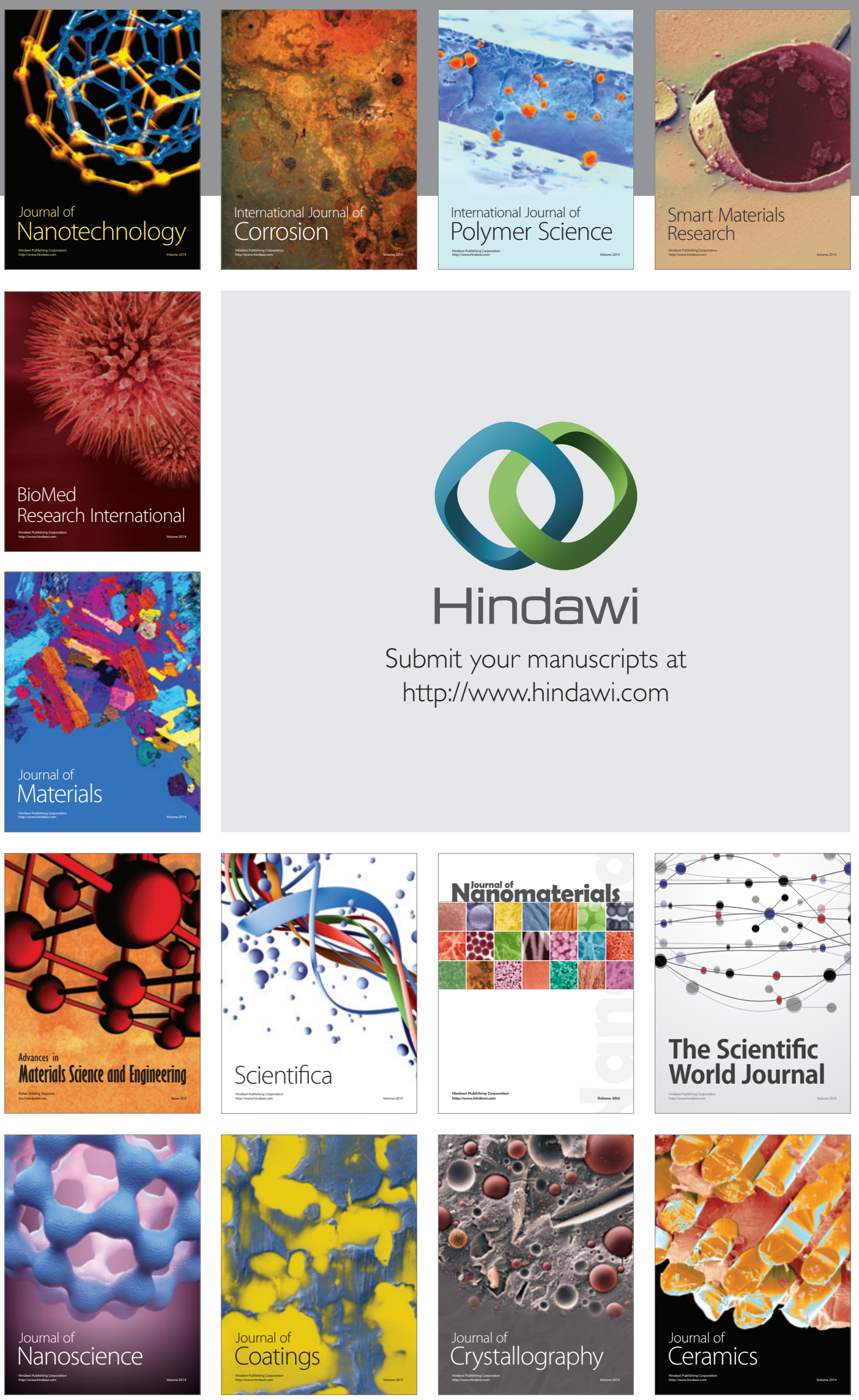

The Scientific World Journal

Submit your manuscripts at

http://www.hindawi.com

\section{World Journal}

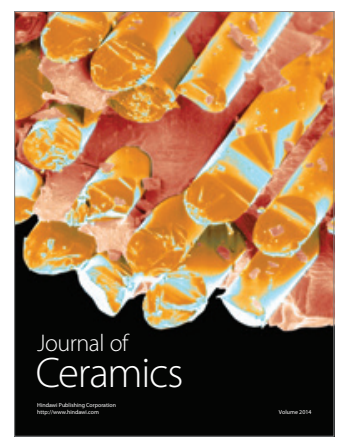

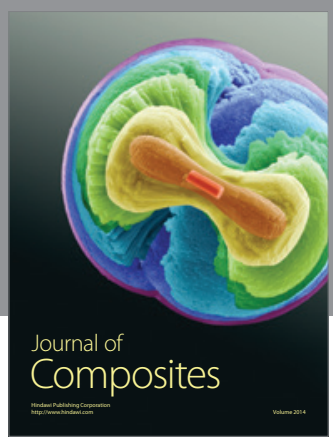
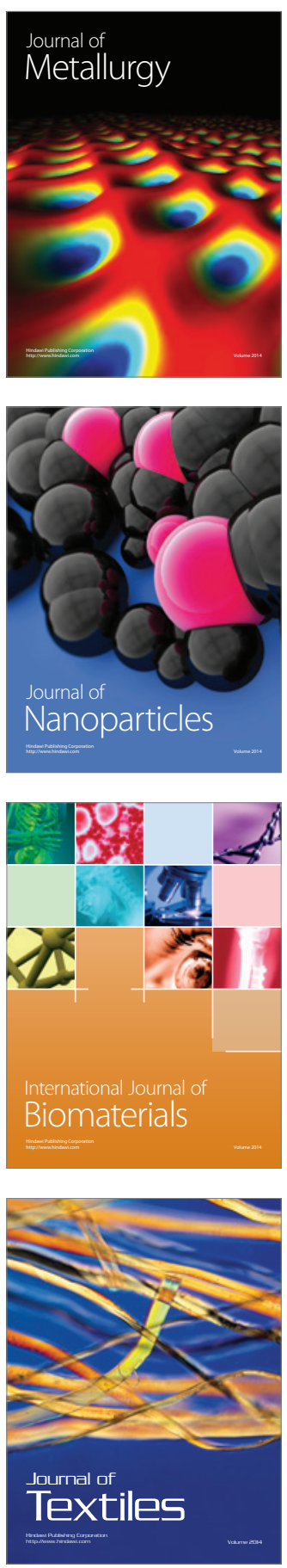